\title{
Evaluation of response methods for the localization of nearby objects
}

\author{
DOUGLAS S. BRUNGART \\ Massachusetts Institute of Technology, Cambridge, Massachusetts \\ and Air Force Research Laboratory, Wright-Patterson Air Force Base, Ohio \\ and \\ WILLIAM M. RABINOWITZ and NATHANIEL I. DURLACH \\ Massachusetts Institute of Technology, Cambridge, Massachusetts
}

\begin{abstract}
Four response methods for indicating the perceived locations of nearby objects were evaluated: the direct-location (DL) method, where a response pointer is moved directly to the perceived location of the target; the large-head (LH) and small-head (SH) methods, where the pointer is moved to the target location relative to a full-scale or half-scale manikin head; and the verbal report (VR) method, where the spherical coordinates of the target location are indicated verbally. Measurements with a visual target indicated that the DL method was relatively unbiased and considerably more accurate than the other methods, which were all roughly equivalent. Correcting for bias improved accuracy in the $\mathrm{LH}, \mathrm{SH}$, and VR responses, but not to the level of the uncorrected DL responses. Replacing the visual target with an acoustic stimulus approximately doubled the errors with the DL response but indicated similar performance in the front and rear hemispheres. The results suggest that DL is the most appropriate response method for close-range localization experiments.
\end{abstract}

In traditional psychoacoustic localization studies, subjects have been asked to estimate either the direction of a sound source or its distance. The perceived distance and direction of a sound source have been simultaneously measured only in a few limited cases. In planning an experiment to examine localization ability for nearby sound sources (less than $1 \mathrm{~m}$ away from the listener), a response method was required that would accurately measure localization accuracy in azimuth, elevation, and distance. Since some targets would be extremely close to the subject ( $5 \mathrm{~cm}$ or less from the head), a subject-based coordinate system was used to provide a consistent basis for expressing locations relative to the head in terms of spherical coordinates. The subject would be required to respond to source locations behind the head and out of the visual field, so a response method that would be consistent for locations in front and behind the listener was desired. Furthermore, since a large number of trials was required, the response method had to be relatively fast. Although our primary motivation was to develop a response method for an auditory localization experiment, these same requirements could apply to any experiment in which a subject is required to report a perceived location within $1 \mathrm{~m}$ of the head (e.g., visual or proprioceptive ex-

The facilities and most of the equipment for these experiments were provided by the Human Effectiveness Directorate, Armstrong Laboratory (AFRL/HECB), Additional support was provided by AFOSR Grant F49620-96-1-0202. Correspondence concerning this article should be addressed to D. S. Brungart, AFRL/HECB, 2610 Seventh Street, WPAFB, OH 45433-7901 (e-mail: douglas.brungart@he.wpafb.af.mil). periments). No response method found in the literature seemed adequate.

Four response methods were tested: direct location (DL), large-head (LH) transformation, small-head (SH) transformation, and verbal report (VR). In the DL method, the subject moves an electromagnetic position sensor directly to the perceived location. In the $\mathrm{LH}$ and SH paradigms, the subject moves the position sensor to a location relative to a full-sized or half-sized manikin head that matches the perceived source location, relative to his or her own head. In the VR response, the subject simply states the coordinates of the perceived sound in degrees azimuth, degrees elevation, and distance. These four response methods were tested by determining the accuracy of subject responses when the stimulus was a visual target. The results show that the DL method is superior to the other three methods, and that the $\mathrm{LH}, \mathrm{SH}$, and VR methods are roughly comparable.

\section{BACKGROUND}

In directional localization experiments, researchers have depended on two types of response methods-VR and pointing. Wightman and Kistler (1992) used VRs to collect azimuth and elevation responses. Subjects stated the perceived source azimuth and elevation in degrees, and the investigator typed these coordinates directly into a computer. This procedure has two major drawbacks. First, the verbal response must be correctly interpreted and properly entered by the investigator; as a result, this procedure has a higher chance of error than an automated system, where the control computer can directly read the 
subject's response. Second, the response method is slow; Wightman and Kistler (1992) collected only 2-3 responses per minute, using this technique.

Makous and Middlebrooks (1990) used a head-pointing response method in their directional localization experiments. Subjects wore an electromagnetic head-tracking sensor and responded by pointing their nose in the perceived direction of the source. This method is slightly faster than the VR method (3-4 responses per minute) and eliminates data entry errors. However, head pointing is difficult for locations behind and above the subject, and it complicates methods for immobilizing the subject's head.

Gilkey, Good, Ericson, Brinkman, and Stewart (1995) examined an alternative to these methods called the God's eye location pointing (GELP) method. The subject was seated with a plastic sphere $(20 \mathrm{~cm}$ in diameter) located $22 \mathrm{~cm}$ in front of the subject and approximately $50 \mathrm{~cm}$ below ear level. The subject moved an electromagnetic sensor on the surface of the plastic sphere to the perceived direction of the sound. This method eliminated the need for the subject to speak or to move his or her head and permitted the use of a bite bar to restrict head motion. Furthermore, the subjects never had to move their hands away from the response sphere, so their responses could be made quickly (16-20 per minute). Gilkey assessed the GELP method with two experiments. First, subjects were asked to identify the directions of sound sources; the average angle error was $18.2^{\circ}$. This performance is comparable with the $20^{\circ}$ average error reported by Wightman and Kistler (1992), using VRs. The mean errors in azimuth and elevation were slightly lower than those reported by Makous and Middlebrooks (1990), using head pointing. In a second assessment, the sound source was eliminated; the experimenter simply read verbal coordinates to the subject, who was then asked to respond at that location with the GELP method. This produced mean angular errors of approximately $9^{\circ}$ (vs. $20^{\circ}$ with the sound source). One concern about the GELP system is the possibility that the 20 $\mathrm{cm}$ rigid sphere might generate unwanted reflections that confound the auditory experiment. Overall, however, the GELP system seems to be a significant improvement over head pointing and verbal reporting for giving directional responses.

Without modification, the head-pointing and GELP response methods cannot be used to make distance judgments. Many experiments in audio distance perception have used verbal judgments of distance (Coleman, 1968; Mershon \& Bowers, 1979). Studies that have simultaneously examined directional and distance perception have asked subjects to draw the location of the speaker relative to the listener on a sheet of paper (either in azimuth and distance or in both azimuth and distance and elevation and distance; Butler, Levy, \& Neff, 1980). Gilkey suggested using a wire-frame sphere model in the GELP procedure, to allow subjects to place the response sensor inside the sphere to indicate distance, but did not test this procedure. For a true three-dimensional localization experiment, drawing responses on paper will be slow and will also require additional time to digitize. Verbal report may be adequate, albeit slow, but no data are available on its accuracy in three dimensions.

Four response methods that were potentially appropriate for collecting three-dimensional locational responses at distances less than $1 \mathrm{~m}$ were chosen for evaluation in a visual experiment.

In the DL method, a subject simply moves an electromagnetic position sensor directly to the location of the visual target. The location of the target and the response can be measured by using a spherical coordinate system based on the location of each subject's ears and nose, as is described in the Appendix. A priori, this appears to be a natural response, since no mental transformation of the target location is required and subjects can use their own anatomical reference points. Soechting and Flanders (1989) examined the accuracy of pointing to the remembered locations of visual targets with the tip of the finger. They found that, when pointing in darkness, estimates of direction were quite accurate but that distance was underestimated. The root-mean square vector magnitude error from the tip of the finger to the actual target location was $11.7 \mathrm{~cm}$. Surprisingly, performance did not improve when pointing with the fingertip in a lighted room but did improve substantially when subjects used a pointer, instead of the fingertip, to indicate the target location. Foley (1977) also conducted an experiment that compared the accuracy of visual distance perception when using fingertip pointing and VR and found that the standard deviation was significantly lower in the pointing condition. Although the DL response has many desirable properties, no data are available on the accuracy of pointing to targets that are outside the visual field. It is possible that pointing accuracy will decrease rapidly for sources behind the subject, both because no visual feedback is available and because such locations can be difficult to reach even with a pointer. A different technique that would be roughly equivalent for locations in front and behind the listener was desired.

The LH transformation technique requires the subject to remember the location of the target relative to his or her own head and to move an electromagnetic position sensor to that same location relative to a full-sized Styrofoam manikin head. It was hoped that the anatomical features of this head would allow better judgments of distance and direction than would the perfect sphere used for responses in the GELP technique. Because the experiment limited all target locations to the right hemisphere, the head was placed in profile, facing to the right of the subject; thus, response locations in front, to the right, and behind the head were equally accessible. In order to allow responses at distances up to $1 \mathrm{~m}$ on the right side, the head was placed $1 \mathrm{~m}$ away from the subject. Response locations were 
a) Source Placement

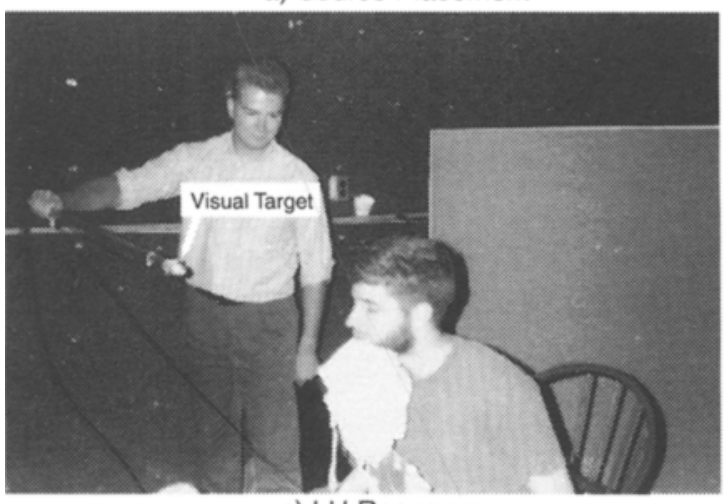

c) LH Response

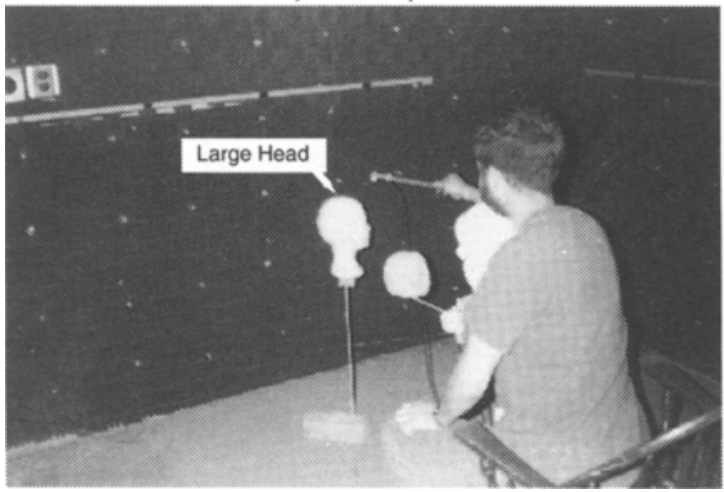

b) DL Response

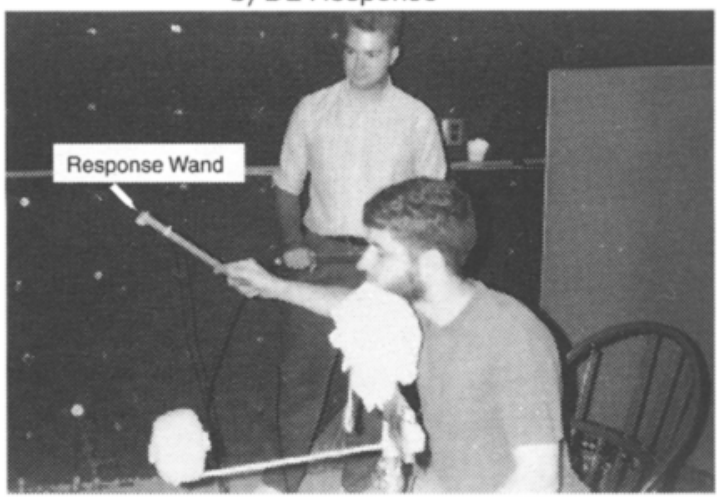

d) SH Response

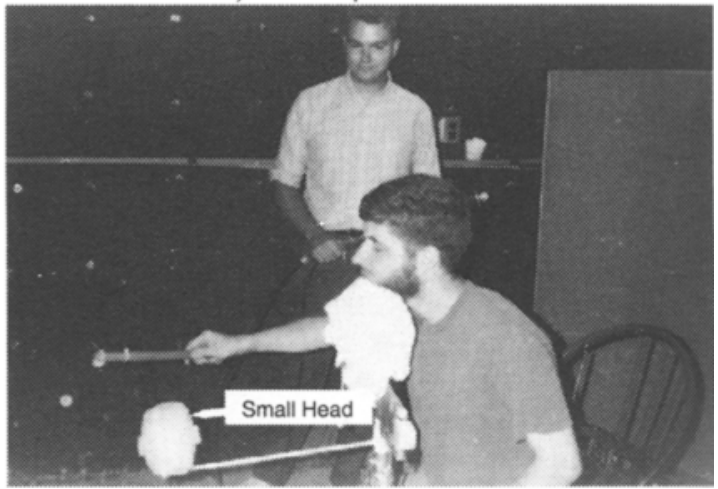

Figure 1. Setup and procedure used for the pointing experiments. The experimenter first places the sensor on the target wand at a random location in the front right quadrant of the subject, makes sure the subject has seen the location, and presses a response button to have the control computer record that location (panel a). The target is then moved away, and the subject estimates the location of the target by four methods: moving the sensor on the response wand to the location of the target (DL; panel b), moving the sensor to the appropriate location relative to the large manikin head (LH) corresponding to the location relative to his or her own head (panel c), moving the sensor to the location relative to the small manikin head (SH; panel d), and verbally reporting the azimuth, elevation, and distance of the source. Note that a soft cloth is draped over the chinrest to enhance the comfort of the subjects.

recorded by using a coordinate system based on the locations of the ears and nose of the manikin head (see the Appendix).

In the SH transformation technique, the full-sized Styrofoam manikin head used in the LH technique is replaced with a half-scale soft foam head. When making a response, the subject moves a position sensor to the location relative to the manikin head corresponding to the location of the target relative to his or her own head, as in the LH response. The major difference between the two responses is that the subjects are expected to scale distance by a factor of one half in the SH response, allowing the manikin head to be placed closer to the body.

In the VR technique, the subjects were familiarized with the spherical coordinate system and were asked to give verbal estimates of the azimuth and elevation (in degrees) and distance (in inches) of the target. The VR response is very similar to that used by Wightman and Kistler (1989) in their localization studies.

\section{EXPERIMENT 1}

The first experiment was designed to compare the basic accuracy of the four response methods when there was little uncertainty about the actual target location. A visual target was used, and source locations were limited to the subject's field of vision. The results compare the fundamental accuracy of each of the four response methods.

\section{Method}

\section{Apparatus}

Figure 1 shows the overall setup used in the experiment. The experiment was conducted in a large, normally lighted listening booth. The four walls and ceiling of the booth were covered with acoustic foam, and the floor was carpeted. The subjects were seated on a wooden chair near the center of the room and were asked to immobilize their heads with the help of a chinrest constructed from plastic pipe mounted on a heavy base plate. The top of the chinrest consisted of a Lucite block and two plastic screws covered by a soft cloth; the screws provided a reference point that allowed the sub- 
Table 1

Response Order in Each Block for Each Subject

\begin{tabular}{lllll}
\hline Subject & Block 1 & Block 2 & Block 3 & Block 4 \\
\hline L.C.L. & VLDS & LSVD & DVSL & SDLV \\
W.R.D. & LSVD & SDLV & VLDS & DVSL \\
L.A.R. & DVSL & VLDS & SDLV & LSVD \\
K.R.J. & SDLV & DVSL & LSVD & VLDS \\
\hline
\end{tabular}

Note- $V$, verbal report; $S$, small-head response; $L$, large-head response; $\mathrm{D}$, direct location.

jects to maintain a consistent head position throughout each block of trials.

A half-scale model of a human head was mounted on the chinrest in front of and below the subject's head. The model was fabricated from soft packing foam and was roughly $10 \mathrm{~cm}$ wide at the interaural axis. Although a crude replica, the foam head exhibited most of the basic features of the human head. Eye sockets and a prominent chin were carved into the solid foam, and a nose, ears, and lips were fashioned separately and glued onto the head with rubber cement. A wooden dowel was affixed to the bottom of the head and attached to the chinrest. This suspended the manikin head $25 \mathrm{~cm}$ below and $50 \mathrm{~cm}$ in front of the subject's chin. The head was in profile when viewed by the subject and was facing to the subject's right side.

A full-sized replica of a human head was also positioned in front of the subject's chair. This manikin head was made of Styrofoam and was purchased from a wig shop. The features of this head included ears, eyes, nose, mouth, and neck. The full-sized head was relatively small for a human, with an interaural axis length of approximately $16 \mathrm{~cm}$. The head was mounted on a vertical dowel rod in a wooden base and was placed in profile facing to the subject's right) $100 \mathrm{~cm}$ in front and $25 \mathrm{~cm}$ below the subject's chin.

A Polhemus 3SPACE tracker was used to record stimulus and response positions. The source of the tracker was attached to the chinrest assembly approximately $20 \mathrm{~cm}$ below the subject's chin. One sensor was mounted on the end of a wooden rod, $33 \mathrm{~cm}$ long and $2 \mathrm{~cm}$ in diameter, that was used by the subject to make responses. The other sensor was attached to the end of a clear plastic tube, $58 \mathrm{~cm}$ long and $3 \mathrm{~cm}$ in diameter, which the experimenter used to place the stimuli. The experimenter also used a small hand-held switch to signal when stimulus and response positions should be recorded by a control computer. The Polhemus system is capable of measuring the three Cartesian coordinates of the sensors relative to the source within $0.5 \mathrm{~cm}$ in each dimension at distances up to $90 \mathrm{~cm}$ and with slightly degraded accuracy at greater distances.

\section{Calibration}

Prior to each block of trials, the subject was asked to find a comfortable position in the chinrest and to immobilize his or her head. Then the Polhemus system was calibrated, using nine reference positions. Specifically, the Cartesian coordinates of the left ear, right ear, and tip of the nose were recorded for the subject's head, the large manikin head, and the small manikin head. As is discussed in the Appendix, these reference locations were used to establish the coordinate systems used for each of the three heads throughout the block of trials.

\section{Stimulus}

The target location in each trial was indicated by the position of a visual pointer. This pointer, a black Poihemus head-tracking sensor (approximately cube shaped, $2 \mathrm{~cm}$ in each dimension) mounted above the end of the clear plastic source wand, was moved by the experimenter to a random position in the front right quadrant of the subject at the beginning of each trial. To determine a random location for the target, the experimenter rolled three fair six-sided dice.
One die designated the approximate azimuth of the target (ranging from $0^{\circ}$ for a one to $90^{\circ}$ for a six), one die designated the approximate elevation (ranging from $+60^{\circ}$ for a one to $-60^{\circ}$ for a six), and one die designated the approximate distance $(15 \mathrm{~cm}$ for a one to $100 \mathrm{~cm}$ for a six).

Once the target was in position, the experimenter pressed a switch, and the computer recorded the coordinates of the target sensor. Two consecutive measurements of target location were made, and the distance between the two measurements was used to ensure that the target was stationary when the switch was pressed. If the target moved more than $1.3 \mathrm{~cm}$ between the measurements, a warning tone alerted the experimenter to place the target again. If the target did not move, a different tone alerted the experimenter to move the target away and also informed the subject to begin his or her responses.

\section{Responses}

In each trial, the subjects estimated the location of the visual target. using four different response methods-DL, LH, SH, and VR, described in the previous section. In the DL, LH, and SH responses, the subject moved the Polhemus sensor (a tan-colored cube approximately $2 \mathrm{~cm}$ in each dimension) mounted above the tip of the response wand to the appropriate location corresponding to the location of the target relative to his or her own head. Once the response sensor was in place, the experimenter pressed the response switch, and the coordinates of the response (relative to the manikin head) were recorded. Note that, with the half-scale head, the subjects scaled distance down by a factor of two, and the Cartesian coordinates of the response (relative to the small head) were doubled, to allow direct comparison with the other methods.

The VR response did not require the use of the response wand. The subject simply stated the azimuth, elevation, and distance of the target, and these were entered into the control computer by the experimenter.

\section{Subjects and Response Ordering}

Two male and 2 female volunteer subjects, ranging in age from 21 to 26 years, participated in the experiment. Each subject engaged in four blocks of 30 trials each, for a total of 120 trials per subject. Each block of trials lasted approximately $45 \mathrm{~min}$, and the subjects were allowed 15 -min breaks between blocks. Subjects L.C.L. and L.A.R. completed all four blocks in 1 day. Subjects K.R.J. and W.R.D. completed two blocks on each of 2 days.

The response order used in each of the four experimental blocks by each subject is shown in Table 1 .

These sequences were selected to minimize the effects of response order on the overall results according to the following criteria.

1. The position of each response method was different in every block. Each of the four response methods occurred first in one block, second in one block, third in one block, and fourth in one block.

2. No pairs of consecutive response methods were repeated across blocks. The response immediately following a particular response type was different in each of the four blocks.

3. The same four response orders were used for each subject's four blocks of trials, but the order of the blocks was different for each subject.

\section{Raw Data}

Figures 2-4 show the stimulus-response pairs in azimuth (Figure 2), elevation (Figure 3), and log distance (Figure 4), respectively, for each subject and each response method. In each panel, correct (i.e., veridical) responses are indicated by a solid line. From the raw data shown in the left panels of these figures, three observations can be made. First, there are substantial differences between the accuracy of the response methods in each dimension. In azimuth, for example (Figure 2), the spread of responses is much greater for the LH method than for any other response method. Second, biases 
Raw Data

Individual Bias Correction

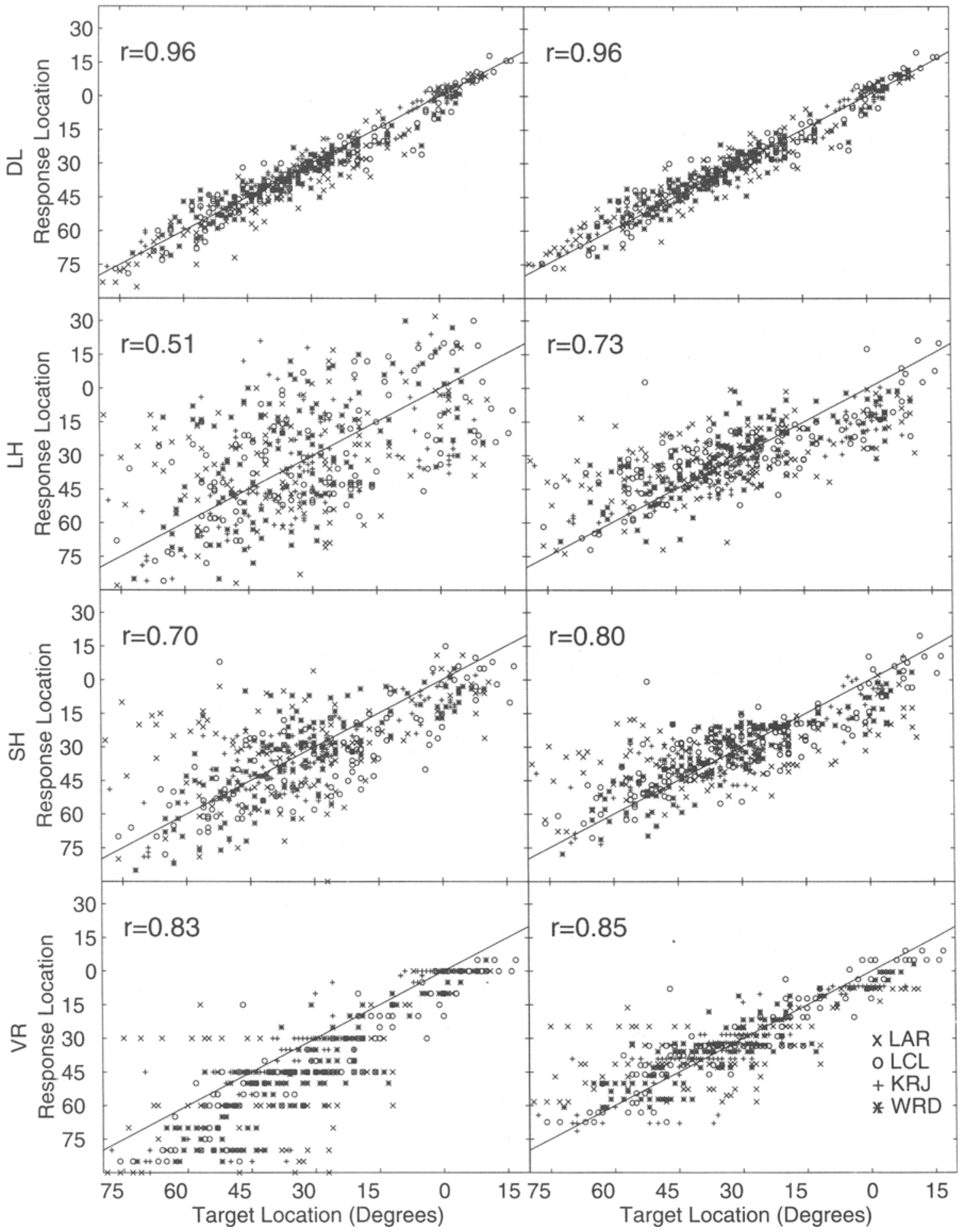

Figure 2. Stimulus-response pairs for azimuth. Each row shows results for a different response method (as labeled). Raw data are plotted in the left panels; data corrected for individual response biases are plotted in the right panels (see the text for details). Different symbols are used to represent the responses of each subject. The solid line indicates correct responses. Note that the responses for verbal report are quantized (along the ordinate), representing subject biases in favor of particular response values. The azimuths range from $-90^{\circ}$ directly to the right of the subject to $0^{\circ}$ directly in front of the subject. DL, direst location; LH, large-head method; SH, small-head method; VR, verbal report. 


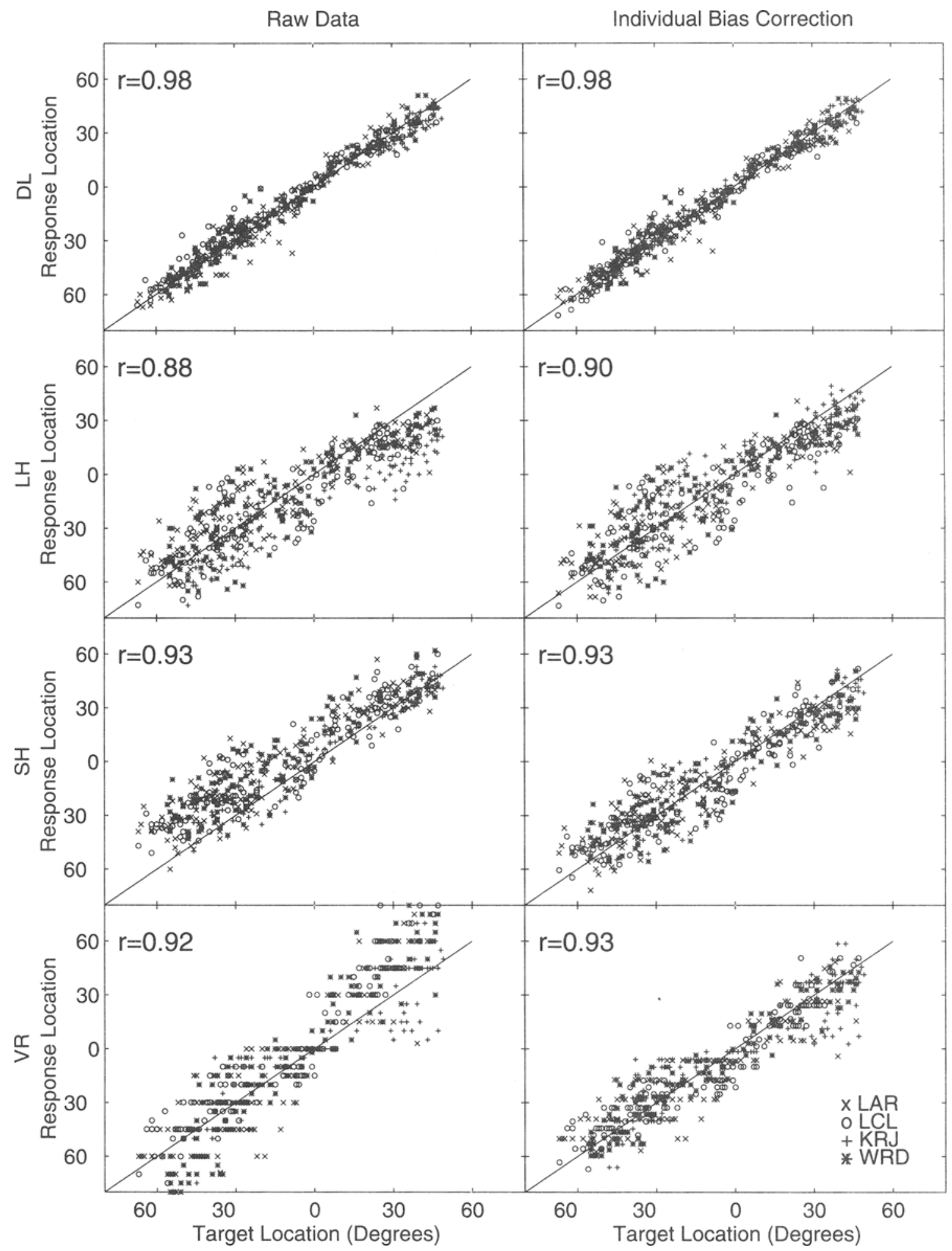

Figure 3. Stimulus-response pairs for elevation. Other details as in Figure 2. Elevations range from $-90^{\circ}$ directly below the subject to $90^{\circ}$ directly above the subject. DL, direct location; LH, large-head method; SH, small-head method; VR, verbal report. 


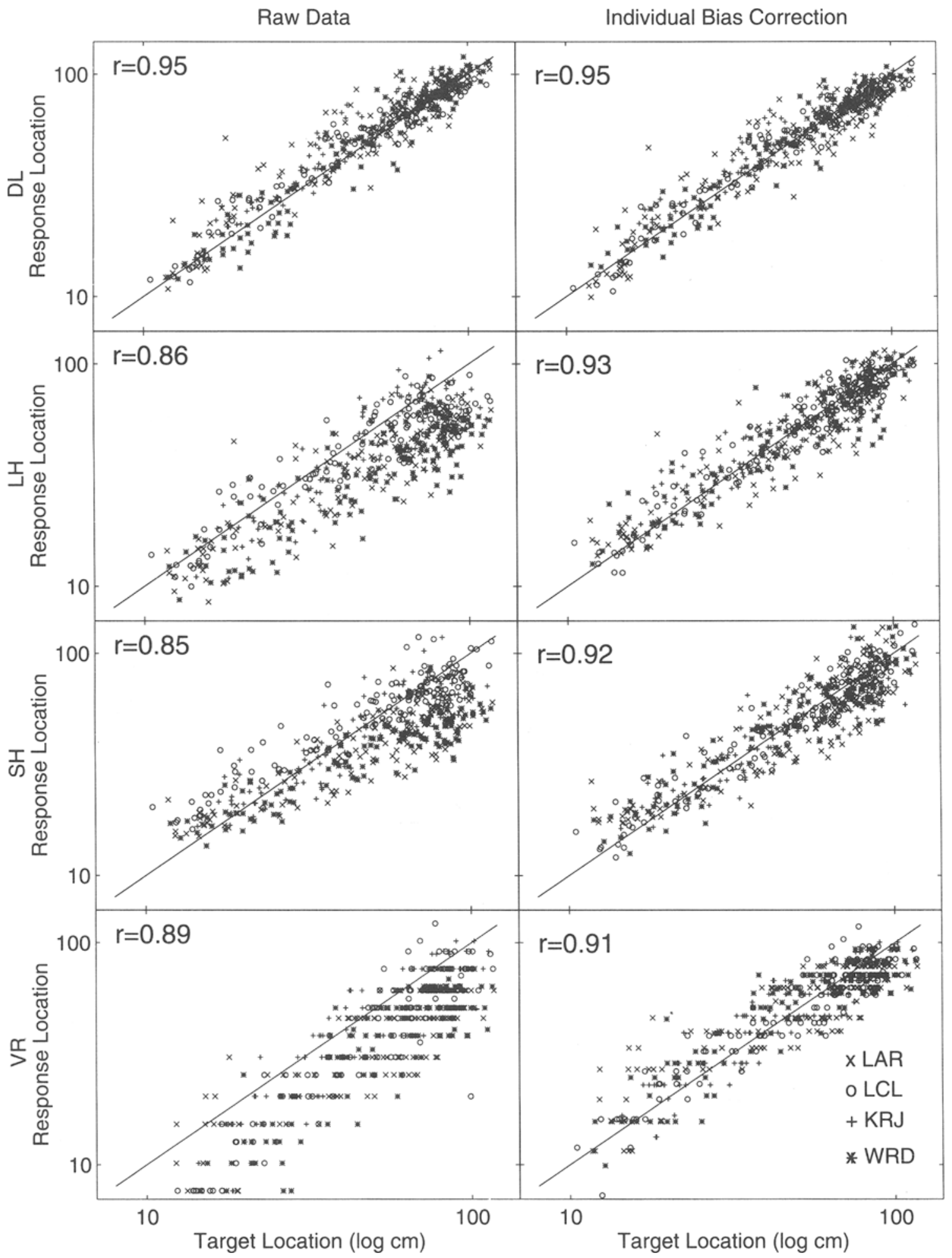

Figure 4. Stimulus-response pairs for distance. Other details as in Figure 2, but for distance rather than azimuth. Distances range from 10 to $100 \mathrm{~cm}$ from the center of the head. DL, direct location; LH, large-head method; SH, small-head method; VR, verbal report. 
Table 2

Mean Errors (With Standard Deviations)

for Each Response Method

\begin{tabular}{|c|c|c|c|c|c|c|c|c|}
\hline \multirow[b]{2}{*}{ Error Type } & \multicolumn{2}{|c|}{ DL } & \multicolumn{2}{|c|}{ LH } & \multicolumn{2}{|c|}{ SH } & \multicolumn{2}{|c|}{ VR } \\
\hline & $M$ & $S D$ & $M$ & $S D$ & $M$ & $S D$ & $M$ & $S D$ \\
\hline Azimuth & $-1.8^{\circ}$ & 5.2 & $0.5^{\circ}$ & 22.1 & $-1.8^{\circ}$ & 15.1 & $-9.6^{\circ}$ & 12.6 \\
\hline Elevation & $0.5^{\circ}$ & 5.8 & $-4.9^{\circ}$ & 13.9 & $10.6^{\circ}$ & 11.6 & $4.7^{\circ}$ & 15.9 \\
\hline Distance & $7.6 \%$ & 19.9 & $-21.8 \%$ & 23.7 & $-9.6 \%$ & 26.1 & $-26.2 \%$ & 22.4 \\
\hline Angle & $6.0^{\circ}$ & 4.2 & $21.7^{\circ}$ & 9.5 & $17.6^{\circ}$ & 9.4 & $18.7^{\circ}$ & 10.6 \\
\hline Vector length & $21 \%$ & 16 & $48 \%$ & 15 & $41 \%$ & 18 & $45 \%$ & 16 \\
\hline
\end{tabular}

Note--DL, direct location; LH, large-head; SH, small-head; VR, verbal report.

are evident in that the responses are often clustered away from the correct response, particularly with the $\mathrm{LH}, \mathrm{SH}$, and VR methods. For example, in distance with the VR method (Figure 4), the responses cluster below the solid line, indicating a bias to underestimate the target distance. Third, and of greatest significance, the DL method has the least response variability and the least bias of the four methods, with responses clustered closely around the correct response in azimuth, elevation, and distance.

\section{Results}

Five measures of response accuracy were used to quantify the results from the four response methods. These measures (Table 2) summarize the mean errors and standard deviations for the following error parameters.

1. The signed error in azimuth: the difference between the azimuth location of the response and the azimuth location of the target. Recall that $-90^{\circ}$ is directly right of the subject and all the targets were on the right side, so a negative value for this error parameter implies that the response was more lateral than the target.

2. The signed error in elevation: the difference between the elevation location of the response and the elevation location of the target. Note that $90^{\circ}$ is directly above the subject, so a negative value of signed elevation error implies that the response is below the target.

3 . The signed percentage distance error: the percent difference between the target distance and the response distance

$$
\left(\frac{r_{\text {response }}}{r_{\text {target }}}-1\right) \cdot 100 \% .
$$

A negative value indicates that the subjects have underestimated distance.

4. The angle error: the magnitude of the angle of the arc between the target and the response locations on a great circle centered at the origin. Note that this error depends on both the stimulus azimuth and elevation and the response azimuth and elevation.

5. The vector length error: the ratio of the length of the vector going from the location of the response to the location of the target divided by the distance from the center of the head to the target, expressed as a percentage. The vector length error includes both directional and distance components and measures overall performance in the task.

These five errors can be divided into two categories. The signed azimuth, elevation, and percentage distance errors can be positive or negative, according to the direction of the error. The mean value of these quantities represents the bias of the responses, whereas the standard deviation measures consistency. In contrast, the angle and vector length errors are strictly positive error measurements. The mean values of these parameters represent the total error, including any response bias and the spread of the responses around the mean, whereas the standard deviations are useful primarily for evaluating the significance of changes in the mean value. When responses are unbiased or when bias is removed, as will be described in the next section, the mean angle and vector length errors measure only the spread of responses and become similar to the standard deviations of the signed errors.

Note that all of these errors are based on an underlying assumption that the actual location of the target is correctly perceived and that any difference between the location of the stimulus and the location of the response represents an error in the response. Of course, some error may also be a result of incorrect perceptions of the response location. If such misperceptions affect all four response measures equally, they are largely irrelevant to this comparative study. However, it should be recognized that the DL method might have a distinct advantage over the other methods if there are systematic discrepancies between an object's apparent visual position and its true position, since the response wand in the DL condition could be placed so that its apparent visual position is the same as that of the previously observed stimulus. Since the response error cannot be isolated completely from errors in the perceived location of the stimulus, there is no way to eliminate this possible advantage from the DL response. However, any systematic visual misperceptions would primarily impact the bias measures (mean azimuth, elevation, and distance errors) and not the variability measures (standard deviations of azimuth, elevation, and distance and angle error). Some evidence that our assumption about veridical perception of source location has not materially affected our results, based on the elimination of systematic biases from the response data and on the results of an experiment with a nonvisual stimulus, will be presented later.

As can be seen in Table 2, the azimuth response is essentially unbiased, except in the VR paradigm, where an average bias of almost $10^{\circ}$ toward the right side occurs. For elevation, the largest bias is with the $\mathrm{SH}$ paradigm, where the subjects, on average, responded more than $10^{\circ}$ 

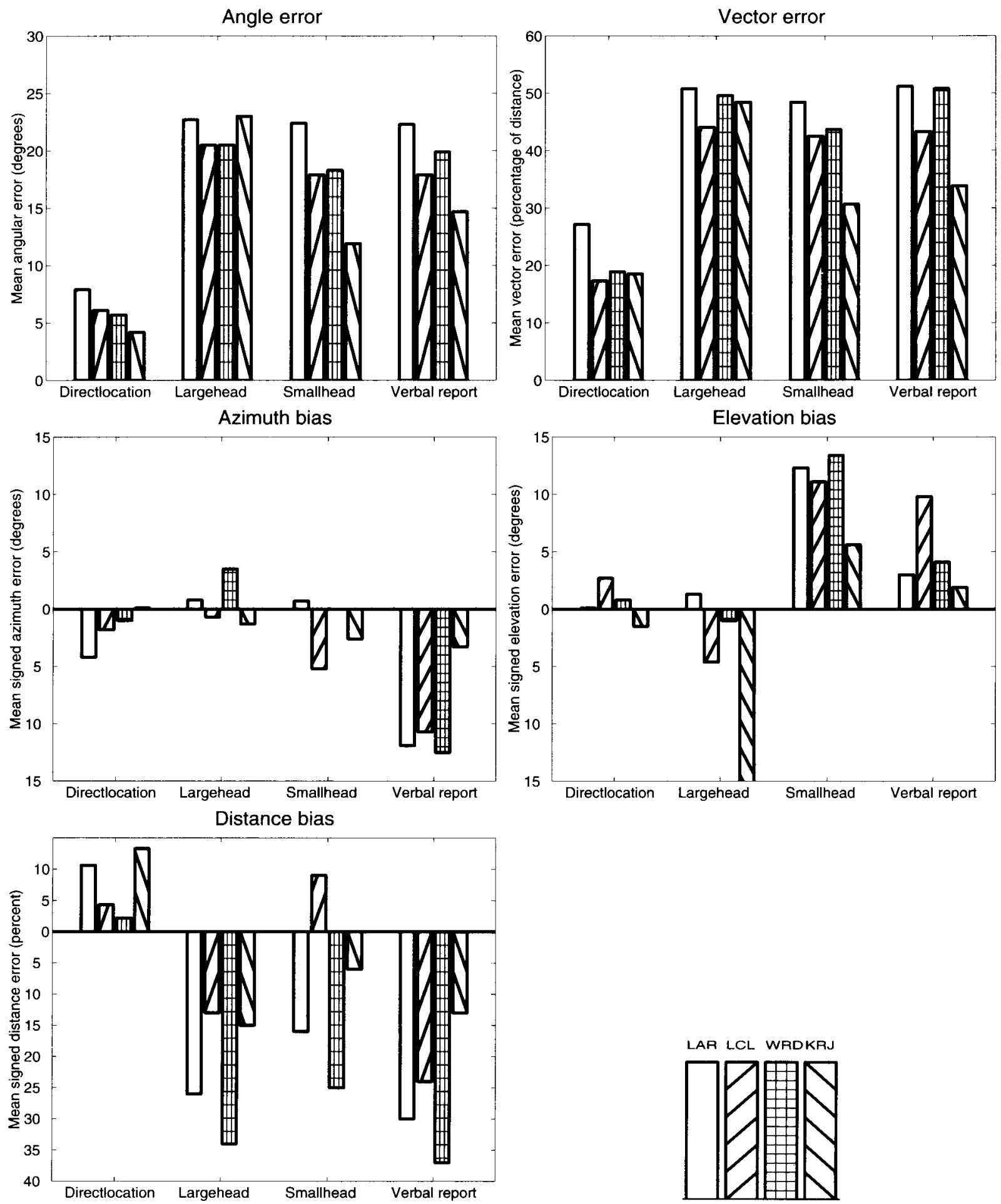

Figure 5. Mean errors for each subject and each response method.

above the true target location. The other biases in elevation were less than $5^{\circ}$ in magnitude. In both azimuth and elevation, DL had less than $2^{\circ}$ of bias. The signed distance errors show that, on average, the subjects overestimated distance in the DL response but underestimated distances when using the other response methods.
For angle error, an analysis of variance (ANOVA) on the raw data shows that the main effect of response method is significant $[F(3,1650)=425.12, p<.0001]$. Pairwise $t$ tests (at the $p<.01$ level) show that the DL method is the best directional response method (mean angle error = $6.0^{\circ}$ ), the SH and VR methods are not significantly dif- 
Table 3

Mean Location of the Visual Target for the Stimuli Used With Each Subject

\begin{tabular}{lccc}
\hline Subject & Azimuth $\left(^{\circ}\right)$ & Elevation $\left(^{\circ}\right)$ & Distance $(\mathrm{cm})$ \\
\hline L.A.R. & -33.8 & -15.2 & 54.5 \\
L.C.L. & -29.3 & -10.8 & 57.8 \\
W.R.D. & -32.4 & -12.1 & 57.4 \\
K.R.J. & -31.8 & 0.6 & 56.2 \\
\hline
\end{tabular}

Note-Values are given on the basis of the coordinate system determined for each subject. With the exception of elevation for subject K.R.J., the mean locations are roughly constant across subjects.

ferent (mean errors $=17.6^{\circ}$ and $\left.18.7^{\circ}\right)$, and the LH method is the worst (mean error $=21.7^{\circ}$ ).

As the final measure of accuracy, the vector length error shows again that the DL method is superior to the other pointing methods (mean error $=21 \%$ ), followed by the SH method (mean error $=41 \%$ ), then the VR method (mean error $=45 \%$ ), and finally the LH method (mean error $=48 \%$ ). The DL method is clearly best, and the differences among the other three methods, although small, are also significant (one-tailed $t$ test, $p<.01$ ).

The overall results can be summarized as follows.

1. The DL response method is greatly superior to all other methods and is essentially unbiased in direction.

2. The LH, SH, and VR responses all exhibit some type of directional bias (in azimuth, elevation, or both), and all underestimate distance.

3. The overall angle errors for the $\mathrm{LH}, \mathrm{SH}$, and $\mathrm{VR}$ methods are all large, although the $\mathrm{LH}$ method is slightly (but significantly) worse than the other two methods.

\section{Intersubject Variability (Raw Data)}

Although there were some differences in performance among the 4 subjects, they performed quite similarly, in general (see Figure 5). For both the angle (great circle) error and the vector length error (the top two panels of Figure 5), all 4 subjects exhibited the best performance (smallest mean error) with the DL method. For the other response methods, 3 of the 4 subjects performed similarly. The 4th subject (K.R.J.) showed significantly better performance with the VR and SH methods than with the LH method.

The biases in each response method were also generally similar among the subjects (the bottom three panels of Figure 5). For every response method and every response dimension, at least 3 of the 4 subjects exhibit the same general bias trend (negligible bias, positive bias, or negative bias). In three cases, subject K.R.J. deviates from the general bias trend. K.R.J. has a much smaller bias in azimuth than the other subjects in the VR method, and his mean elevation response is significantly below those of the other subjects with the LH and SH methods. Since the other subjects exhibit small elevation biases for the $\mathrm{LH}$ response and large positive elevation biases for the SH response, K.R.J.'s unique trends make him the least biased subject with the SH method and the most biased subject with the LH method. These differences in bias help explain why K.R.J.'s mean angle and vector length errors (which include bias effects) are smaller with the VR and SH methods than with the LH method.

A difference in head positioning during the experiment may explain the unusual elevation response biases exhibited by subject K.R.J. An examination of the orientation of the subjects' heads during calibration indicates that K.R.J.'s head was tilted slightly down (i.e., his nose was slightly lower than his interaural axis), whereas the other subjects' heads were tilted slightly up. The effect of this tilt is clearly seen in Table 3 , which shows the mean stimulus target locations for the 4 subjects. Whereas the average target azimuths and distances were similar for the 3 subjects, the average target elevation for K.R.J. was $13.3^{\circ}$ higher than the average target elevation for the other 3 subjects. This difference suggests that K.R.J.'s head was tilted slightly down during the experiment and that the experimenter did not correct for this tilt when positioning the visual target. If a subject's internal representation of the target location did not account for the tilt of his head, he would position the target sensor at a lower elevation, relative to the manikin head, when his head was tilted downward than when his head was tilted upward, whereas the response bias with the DL method would be unchanged. The bias trends in Figure 5 are consistent, therefore, with K.R.J.'s head having been tilted downward and the other subjects' heads tilted upward, although it is not clear why the VR response is apparently unaffected.

\section{Bias Correction}

Overall correction. In every response method except DL, the subjects generally performed quite poorly. Angle errors were close to $20^{\circ}$, and vector length errors were approximately $45 \%$. In part, these overall errors were caused

Table 4

Bias Correction Parameters and Correlation Coefficients

\begin{tabular}{|c|c|c|c|c|c|c|c|c|c|c|c|c|}
\hline \multirow[b]{2}{*}{ Location } & \multicolumn{3}{|c|}{ DL } & \multicolumn{3}{|c|}{$\widetilde{\mathrm{LH}}$} & \multicolumn{3}{|c|}{$\mathrm{SH}$} & \multicolumn{3}{|c|}{ VR } \\
\hline & $m$ & $b$ & $r_{\text {corr }}$ & $m$ & $b$ & $r_{\text {corr }}$ & $m$ & $b$ & $r_{\text {corr }}$ & $m$ & $b$ & $r_{\text {corr }}$ \\
\hline Azimuth & 0.97 & 0.9 & .96 & 0.42 & -18.6 & .51 & 0.71 & -8.6 & .70 & 0.69 & -3.2 & .83 \\
\hline Elevation & 1.00 & -0.5 & .98 & 1.05 & 5.5 & .88 & 1.05 & -10.6 & .93 & 0.73 & -5.9 & .92 \\
\hline Distance & 0.98 & 0.0 & .95 & 0.93 & 0.5 & .86 & 1.06 & -0.1 & .85 & 0.70 & 1.1 & .89 \\
\hline
\end{tabular}

Note- $m$ gives the slope and $b$ gives the $y$-intercept of the linear bias corrections. The transformed value of response $x$ in each case is $m x+b$. The correlation ( $\left.r_{\text {corr }}\right)$ between target location and response location is also shown. 


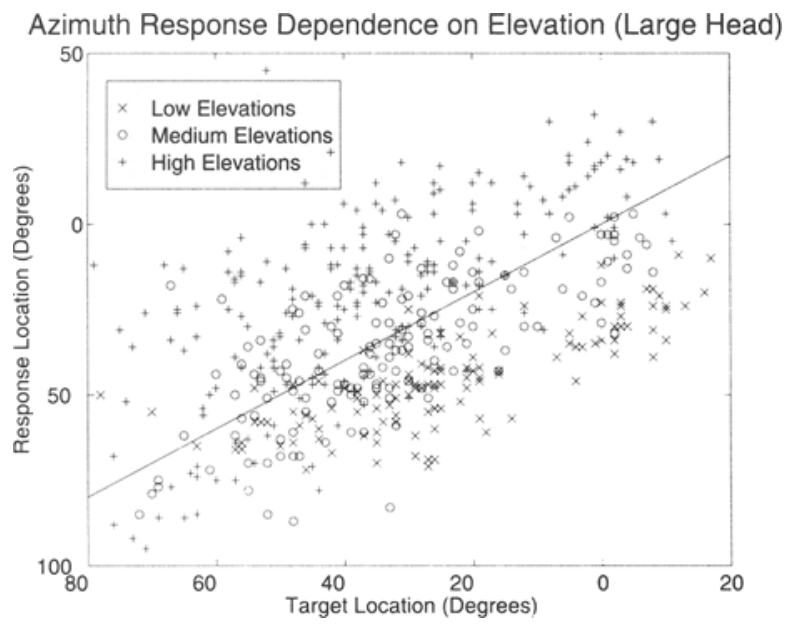

Figure 6. Stimulus and response azimuth for the LH method, with elevation as a parameter. The three symbols represent biascorrected elevation responses less than $-15^{\circ}$ (low), between $-15^{\circ}$ and $15^{\circ}$ (medium), and greater than $15^{\circ}$ (high). Note that the azimuth response has a negative bias at low elevations (with $x$ s falling below the zero-bias diagonal), is approximately unbiased at medium elevations, and is positively biased at high elevations.

by biases in the responses. In the VR condition, for example, responses were, on average, approximately $10^{\circ}$ too far to the right and $26 \%$ too close (Table 2). Each of these biases contributed to the average errors of $17.6^{\circ}$ for angle and $45 \%$ for vector length. If biases were systematic, the accuracy of a given response method could be enhanced by transforming the biased response locations into nonbiased estimates of actual source location. Furthermore, if these biases resulted from a systematic misperception of the location of a visual stimulus, the elimination of these biases would allow a more direct comparison of performance for the DL response (in which the same misperceptions would be made about the placement of the response wand as those that were made in the observation of the stimulus, resulting in an apparently unbiased response) to the other response methods (where these misperceptions would be reflected directly in the response biases).

A linear transformation of each response coordinate was used to compensate for response biases. The coefficients of these linear transformations were found from the linear regression of the actual target coordinates (independent variable) on the response coordinates (dependent variable), separately for azimuth and elevation. The distance transformation was based on the regression of the log of the actual distance on the log of the response distance. The parameters of the overall bias corrections are shown in Table 4.

Elevation-dependent correction. The bias correction can be improved further by exploiting interactions that occur across response dimensions. An analysis of the biases in azimuth, elevation, and distance revealed that response biases for elevation were essentially independent of both distance and azimuth for all four response meth- ods. The response biases in azimuth were independent of distance but were strongly dependent on elevation for the $\mathrm{LH}$ response (see Figure 6) and slightly dependent on elevation for the SH responses. Similarly, the response biases for distance were dependent on elevation for the LH and $\mathrm{SH}$ responses. Since the elevation responses are independent of target azimuth and distance, it is possible to first estimate the target elevation by transforming the elevation response and to then transform the azimuth and distance responses on the basis of this unbiased estimate of elevation. For simplicity, the estimate of elevation was used to divide the response region into three bins-one for corrected elevations below $-15^{\circ}$, a second for elevations from $-15^{\circ}$ to $15^{\circ}$, and a third for elevations above $15^{\circ}$. Linear regressions of target azimuth on response azimuth were performed in each of the three bins, and the resulting coefficients were used for bias correction in azimuth (Table 5). A similar procedure was used to determine the bias correction coefficients for the log of the response distance (Table 6). The elevation-dependent bias correction causes the correlation of stimulus and response azimuth to increase from .51 to .73 for the LH method and from .70 to .77 for the SH method (see Tables 4 and 5) and slightly increases the stimulus-response correlation in distance for each method.

Subject-dependent correction. When the individual subject data were examined, it was found that I subject sometimes deviated from the overall trend (see Figure 5). It is possible to correct the responses for these individual subject biases. This was done in the same way as that for the elevation-based corrections described in the previous section, except that different correction parameters were calculated for each individual subject. This results, of course, in a large set of correction coefficients, and these coefficients will not be shown. The individually corrected data were used to calculate vector length and angle errors for comparison with the nonindividualized biascorrected results. The correspondence between the subject responses when bias corrected for individual differences and the actual target locations is shown in the right panels of Figures 2, 3, and 4. In all cases, the corrected results are clearly superior to the raw data shown in the left panels, with the responses more tightly clustered around the diagonal representing correct responses.

Bias correction summary. The angle and vector length errors were calculated with each level of bias correction (Table 7). The errors decrease to varying degrees

Table 5

Elevation-Dependent Bias Correction Parameters for Azimuth

\begin{tabular}{lcrrrrrr}
\hline & \multicolumn{3}{c}{ LH } & & \multicolumn{3}{c}{ SH } \\
\cline { 2 - 5 } \cline { 6 - 8 } Elevation & $m$ & $b$ & $r_{\text {corr }}$ & & $m$ & $b$ & $r_{\text {corr }}$ \\
\hline$\leq-15^{\circ}$ & 1.11 & 26.6 & .80 & & 0.86 & 6.1 & .83 \\
$\leq 15^{\circ}$ & 0.70 & -6.3 & .75 & & 0.81 & -2.3 & .82 \\
$>15^{\circ}$ & 0.51 & -25.7 & .65 & & 0.66 & -15.6 & .69 \\
$\quad$ Corrected & & & .73 & & & .77 \\
\hline
\end{tabular}

Note-Elevations were corrected for linear bias before sorting into bins. See the text for details. 
Table 6

Elevation-Dependent Correction Parameters for Log Distance

\begin{tabular}{lccccrrr}
\hline & \multicolumn{3}{c}{ LH } & & & SH \\
\cline { 2 - 5 } \cline { 6 - 8 } Elevation & $m$ & $b$ & $r_{\text {corr }}$ & & $m$ & $b$ & $r_{\text {corr }}$ \\
\hline$\leq-15^{\circ}$ & 0.96 & 0.2 & .92 & & 0.96 & 0.1 & .85 \\
$\leq 15^{\circ}$ & 0.89 & 0.6 & .89 & & 1.12 & -0.2 & .89 \\
$>15^{\circ}$ & 0.93 & 0.7 & .89 & & 1.06 & 0.1 & .84 \\
$\quad$ Corrected & & & .91 & & & .88 \\
\hline
\end{tabular}

Note-Elevations were corrected for linear bias before sorting into bins. See the text for details.

as each additional level of complexity is added to the bias correction scheme.

1. The DL response method changes insignificantly with bias correction, indicating that these responses were essentially unbiased without any corrections.

2. The $\mathrm{LH}, \mathrm{SH}$, and VR responses are all improved by bias correction but remain roughly comparable in overall performance.

3. Even with bias correction, the accuracy of the $\mathrm{LH}$, $\mathrm{SH}$, and VR methods remains substantially inferior to that of the DL method. This indicates that the poor performance in these methods, relative to the DL method, is not entirely a result of systematic errors in the visual perception of the stimulus location.

4. The majority of the performance improvement is obtained with the overall and elevation-based bias correction. This joint correction captures more than $80 \%$ of the total improvement in angle error and at least $50 \%$ of the total overall improvement in vector length error. The additional improvement provided by correction for individual subject biases is small.

These results indicate that a linear bias correction can improve the accuracy of the indirect response methods. Furthermore, it appears that a general bias correction scheme chosen for all subjects will work almost as well as a more complex scheme based on the individual peculiarities of each subject's responses.

\section{Locational Dependence of Response Errors}

A priori, one might expect the accuracy of the response to depend on the location of the visual target. For example, the angle error might be larger for targets off to the side of the subject than for targets directly in front of the subject. In order to investigate this possibility, the correlation coefficients for the response error and the azimuth, elevation, and distance of the stimulus location were computed. The data were first corrected for bias by using the overall elevation-adjusted bias correction discussed previously. The results (Table 8 ) are the correlation coefficients between the error variable (angle and vector length) and the azimuth, elevation, and distance of the target. The angle errors are essentially uncorrelated with target position: The largest correlation coefficient for angle has a magnitude of .17, and the average magnitude of the correlation coefficient is .09 . The vector length errors are also essentially uncorrelated with elevation and azimuth $\left(\left|r_{\text {corr }}\right| \leq .11\right.$; mean magnitude, .07$)$ but negatively correlated with distance for all four response methods (average, -.28 ). This indicates that the vector length error is not simply proportional to distance and may include a constant term that contributes more to the error ratio at close distances than at far distances. Overall, however, it appears that the specific location of the target within the visual field has little influence on performance.

\section{Memory Effects}

On each trial, the subjects were required to use all four response methods for each stimulus. Although this ensures that exactly the same stimulus set was used with each response method, it presents the possibility that the accuracy of a response could depend on its position in the response sequence. A priori, one expects the subject's mental image of the target location to degrade over time; hence, the first response given after each stimulus might be the most accurate, and the last response the least accurate. In our experiment, four different response orders were used, and each response method was first, second, third, and fourth in an equal number of trials.

As a simple assessment of response order effects, we compared the accuracy of each response method in trials in which it was the first response and trials in which it was the last response (Table 9). The DL response degrades the most when it follows the other response methods. The average DL angle error increased $80 \%$, and the vector length error increased $130 \%$, when DL was the last response versus the first response. For LH responses, the average angle and vector errors also increased significantly, but not as dramatically as for the DL response. None of the other errors increased significantly. A likely explanation for these results is that the DL response relies on explicit memory of the source's visual location and that this memory degrades when one is distracted by making other intervening responses. In contrast, the three indirect response methods require one to encode the location of the source and to mentally transform this location into either verbal coordinates or coordinates relative to the manikin heads. This encoded memory may be less volatile than the explicit visual memory. By analogy to the memory model developed by Durlach and Braida

Table 7

Response Errors for Different Levels of Bias Correction

\begin{tabular}{|c|c|c|c|c|c|}
\hline Error & Method & $\begin{array}{c}\text { No } \\
\text { Correction }\end{array}$ & Overall & $\begin{array}{c}\text { Overall } \\
+ \text { Elevation } \\
\end{array}$ & $\begin{array}{c}\text { Individual } \\
+ \text { Elevation } \\
\end{array}$ \\
\hline \multirow[t]{4}{*}{ Angle } & DL & $6.0^{\circ}$ & $6.0^{\circ}$ & & $5.6^{\circ}$ \\
\hline & LH & $21.7^{\circ}$ & $18.6^{\circ}$ & $16.5^{\circ}$ & $15.4^{\circ}$ \\
\hline & SH & $17.6^{\circ}$ & $14.6^{\circ}$ & $13.6^{\circ}$ & $13.0^{\circ}$ \\
\hline & VR & $18.7^{\circ}$ & $13.2^{\circ}$ & & $12.4^{\prime \prime}$ \\
\hline \multirow[t]{4}{*}{ Vector length } & DL & $20 \%$ & $19 \%$ & & $19 \%$ \\
\hline & LH & $48 \%$ & $46 \%$ & $39 \%$ & $35 \%$ \\
\hline & SH & $41 \%$ & $41 \%$ & $37 \%$ & $33 \%$ \\
\hline & VR & $45 \%$ & $35 \%$ & & $33 \%$ \\
\hline
\end{tabular}

Note-DL, direct location: LH, large-head: SH, small-head; VR, verbal report. 
Table 8

Correlation Coefficients Between

Response Errors and Target Location

\begin{tabular}{llrrrr}
\hline \multicolumn{1}{c}{ Error } & Variable & \multicolumn{1}{c}{ DL } & LH & SH & VR \\
\hline Angle & distance & -.13 & -.11 & .03 & -.16 \\
& elevation & .02 & .06 & -.14 & .08 \\
\multirow{3}{*}{ Vector length } & azimuth & -.17 & -.08 & -.10 & -.03 \\
& distance & -.27 & -.34 & -.19 & -.32 \\
& elevation & .03 & .02 & -.08 & .06 \\
& azimuth & -.11 & -.10 & -.09 & .09 \\
\hline
\end{tabular}

Note-DL, direct location; LH, large-head; SH, small-head; VR, verbal report.

(1969), the memory model used for DL responses is a trace memory that degrades over time, and that used for the three indirect responses is a context-coded memory that is temporally invariant. Suppose the same encoded version of the source location was used for all three indirect response methods. Then, one would expect the same types of errors in all three methods within a given trial. This hypothesis was tested by examining the correlations between the errors of the three indirect response methods in each trial. Correlation coefficients between the $\mathrm{LH}$ and the SH response errors were found to be very high -.79 for azimuth errors, .78 for elevation errors, and .76 for log-distance errors. Correlation coefficients between the errors of the other response methods did not exceed .47 (and the average of the other correlations was only .31). It is, therefore, likely that the same mental encoding of location was used for both the LH and the SH responses (not surprisingly, considering the similarities between the tasks), but this encoding does not appear to be the same as that used for the VR responses.

\section{EXPERIMENT 2}

Overall, none of the three indirect response methods was found to be particularly attractive. The accuracies of the $\mathrm{LH}, \mathrm{SH}$, and VR responses were similar when (subject-independent) bias correction was applied. Other factors do differentiate the methods, however. The VR method was found to be too slow to be practical (often taking as long as the other three responses combined), and the LH response showed marginally larger angle errors than the other indirect methods. Consequently, the $\mathrm{SH}$ response method was tentatively chosen as the best of the indirect methods. Since the small manikin allows subjects to scale distances down by a factor of two, it is not necessary to place the manikin in profile (as was done in Experiment 1) in order to allow the subjects to reach all locations in the right hemisphere at distances up to $1 \mathrm{~m}$. That is, the head could be placed facing in the same direction as the subject. We hypothesized that this might yield improved performance by simplifying the internal transformations made by the subject, since a $90^{\circ}$ reference frame rotation is eliminated. A simple experiment was designed to test this hypothesis.

\section{Method}

The second experiment was similar to the first experiment, with only a few exceptions. First, testing was performed in an open laboratory space and not in a small listening booth. Second, the responses were restricted to the $\mathrm{SH}$ technique. The small manikin head was no longer attached to the chinrest but was mounted on the vertical stand previously used for the large manikin head. This allowed the small head to be rotated to face either the same direction as the subject or directly to the right of the subject (i.e., in profile, as in Experiment 1).

Four subjects ( 3 male, 1 female, ranging in age from 23 to 30 years) were paid to participate in the experiment. Four blocks of 50 trials were collected from each of the subjects. For 2 of the subjects, the head was in profile for the first and third blocks and was facing forward in the second and fourth blocks. For the other 2 subjects, the opposite ordering was used. Each block took about $20 \mathrm{~min}$, and the subjects were given a break between blocks.

\section{Results and Discussion}

\section{Comparison With Experiment 1}

The data collected with the head in profile were first compared with the results from Experiment 1 for the trials in which the $\mathrm{SH}$ response was the initial response (Table 10). The similarity between the angle biases and the overall angle error (and their associated standard deviations) is striking. In each experiment, the subjects showed a small negative bias in azimuth and a positive bias of approximately $10^{\circ}$ in elevation. In both cases, the overall angle error was near $16^{\circ}$. Only the difference in elevation bias was statistically significant $(p<.05$, using a two-tailed $t$ test).

In contrast, the bias for distance and the mean vector length errors were both significantly smaller in Experiment 2 than in Experiment 1 . This could be a result of reduced response complexity, since the subjects only needed to think about one response, or of variations among the subjects.

The availability of SH response data from a second group of subjects allows us to compare the best overall bias correction of the two groups. As before, this correction was calculated from the linear regression of the target location on the response location. The results (see

Table 9

Accuracy of the First and Last Responses

\begin{tabular}{|c|c|c|c|c|c|}
\hline \multirow[b]{2}{*}{ Error } & \multirow[b]{2}{*}{ Method } & \multicolumn{2}{|c|}{ First Response } & \multicolumn{2}{|c|}{ Last Response } \\
\hline & & $M$ & $S D$ & $M$ & $S D$ \\
\hline \multirow[t]{4}{*}{ Angle } & $\mathrm{DL}^{*}$ & $4.0^{\circ}$ & 3.0 & $7.2^{\circ}$ & 4.8 \\
\hline & $\mathrm{LH}^{*}$ & $19.8^{\circ}$ & 9.5 & $24.8^{\circ}$ & 9.4 \\
\hline & $\mathrm{SH}$ & $17.0^{\circ}$ & 9.2 & $17.2^{\circ}$ & 9.8 \\
\hline & VR & $19.0^{\circ}$ & 10.9 & $19.3^{\circ}$ & 10.9 \\
\hline \multirow[t]{4}{*}{ Vector length } & $\mathrm{DL}^{*}$ & $12 \%$ & 7 & $28 \%$ & 20 \\
\hline & $\mathrm{LH}^{*}$ & $45 \%$ & 14 & $51 \%$ & 15 \\
\hline & $\mathrm{SH}$ & $41 \%$ & 15 & $42 \%$ & 19 \\
\hline & VR & $47 \%$ & 15 & $46 \%$ & 15 \\
\hline
\end{tabular}

Note-The data were not corrected for biases. The ${ }^{*}$ indicates differences significant at the $p<.001$ level (two-tailed $t$ test); no other differences are significant at the $p<.05$ level. DL, direct location; LH, large-head; SH, small-head; VR, verbal report. 
Table 10

Small-Head Results From Experiments 1 and 2

\begin{tabular}{lccccc} 
& \multicolumn{2}{c}{ Experiment 1} & & \multicolumn{2}{c}{ Experiment 2} \\
\cline { 2 - 3 } \cline { 5 - 6 } Error Type & $M$ & $S D$ & & $M$ & $S D$ \\
\hline Azimuth & $-0.8^{\circ}$ & 12.1 & $-3.4^{\circ}$ & 12.7 \\
Elevation* & $12.5^{\circ}$ & 10.5 & $9.7^{\circ}$ & 9.1 \\
Distance $\dagger$ & $-16.5^{\circ}$ & 23.5 & $-0.6^{\circ} \%$ & 21.2 \\
Angle & $16.9^{\circ}$ & 9.1 & $15.6^{\circ}$ & 7.7 \\
Vector length $\dagger$ & $41 \%$ & 15 & $35 \%$ & 15 \\
\hline
\end{tabular}

Note-Comparison of the average response errors for the small-head response in Experiment I, when it was the first response in a trial, to the response errors for the profiled head condition of Experiment 2. The ${ }^{*}$ and ${ }^{+}$indicate differences significant at the $p<.05$ and $p<.001$ levels, respectively (two-tailed $t$ test).

Table 11) are very similar for both experiments. Although the slope of the bias correction in elevation was slightly higher in the first experiment, the corrections are otherwise nearly identical. Interestingly, the optimal bias correction in distance is identical, even though distance accuracy was significantly better in Experiment 2 .

The individual subject biases (not shown) were not as consistent as the average biases for the two groups. Individual biases in azimuth for the $\mathrm{SH}$ response (in both experiments) ranged from $-10.6^{\circ}$ to $2.7^{\circ}$, and the biases in elevation ranged from $5.6^{\circ}$ to $19.7^{\circ}$. Standard deviations for both azimuth bias and elevation bias were $4.9^{\circ}$. Despite these individual differences, the mean results from the two experiments support the idea that the SH response can be improved substantially with a subject-independent bias correction scheme.

\section{Comparison of Profiled and \\ Forward-Facing Small-Head Conditions}

The principal hypothesis motivating Experiment 2 was that the forward-facing condition would yield better performance than the profiled condition. The results clearly invalidate this hypothesis (see Table 12). In fact, the profiled head condition was found to be slightly, but significantly, more accurate in vector length error. Overall, however, the difference between the two manikin orientations appears negligible, and this difference diminishes further when the data are corrected for overall biases.

\section{EXPERIMENT 3}

In Experiments 1 and 2, the DL response was by far the best response method. The SH response, which was suggested as the best alternative to DL, produced angle and vector length errors 2-3 times as large as the DL response, even when corrected for bias. The DL method was so much better than the indirect response methods in the front hemisphere that it seemed possible that it might also be superior in the rear hemisphere, despite the difficulties of reaching behind the body and outside the visual field.

In order to test response accuracy for locations outside the visual field, a different (nonvisual) stimulus was nec- essary. Since the primary intent of these experiments was to evaluate response measures appropriate for a nearfield psychoacoustic experiment, an auditory stimulus was chosen. Although such a stimulus was expected to generate a noisier response estimate than would a visual target, it would allow useful comparisons of the accuracy of DL inside and outside the visual field.

\section{Method}

The major difference between Experiment 3 and the previous experiments is the use of an auditory stimulus instead of a visual stimulus. The stimulus was generated by an acoustic point source, which consisted of a horn driver (ElectroVoice DH1506) fitted to a clear Tygon tube ( $3.2 \mathrm{~m}$ long, with i.d. of $1.3 \mathrm{~cm})$. The tube was fed through the clear plastic source wand, so that its opening protruded slightly $(2 \mathrm{~cm})$ beyond the end of the wand. In this configuration, the sound source is effectively located at the opening of the tube and is relatively nondirectional over a wide range of frequencies (3$\mathrm{dB}$ beam width $=120^{\circ}$ at $15 \mathrm{kHz}$ ). As in the previous experiments, the Polhemus sensor on the source wand was used to measure the location of the source on each trial; a software modification was made to account for the displacement of the source (the end of the tube), relative to the sensor mounted on the wand. The horn driver was connected to a power amplifier (Crown D-75) that, in turn, was connected to a 16-bit digital sound card (Cyber-Audio) in the control computer. This card was used to generate the sound stimulus, which was a band-limited frozen noise signal $(100 \mathrm{~Hz}$ to $15 \mathrm{kHz})$, $125 \mathrm{msec}$ in duration. The noise was filtered to compensate for the irregular frequency response of the point source, resulting in a freefield spectrum that was roughly flat (within $\pm 3 \mathrm{~dB}$ ) across the passband. The amplitude of the source was fixed and was approximately $60 \mathrm{~dB}$ SPL at a distance of $1 \mathrm{~m}$.

The range of stimulus locations was expanded from the front right quadrant to the entire right hemisphere. An approximate azimuth location of the stimulus was still generated by rolling a sixsided die on each trial, but the range was increased from $0^{\circ}$ to $-180^{\circ}$. The subjects were asked to keep their eyes closed while the source was being placed prior to each trial. Then a noise burst was generated at the random target location, and the point source was moved to a neutral position. After the source was moved away, the subjects opened their eyes and gave estimates of the source location with the $\mathrm{DL}$ and SH responses. The subjects used the chinrest to keep their heads stationary throughout the experiment.

Two subjects were tested, I male and 1 female, both of whom had previously participated in Experiment 1. Four blocks of 50 trials were collected from each subject, but a few trials had to be discarded because of head-tracker failures, so a total of 392 trials were usable. Each block of trials took approximately $30 \mathrm{~min}$. In some trials, subjects misperceived sound sources in the front hemisphere as arising from the rear hemisphere, a phenomenon that has been reported frequently by others. Whenever both the $\mathrm{DL}$ and the $\mathrm{SH}$ re-

Table 11

Bias Correction Parameters and

Correlation Coefficients in Experiments 1 and 2

\begin{tabular}{lccccccc} 
& \multicolumn{3}{c}{ Experiment 1} & & \multicolumn{3}{c}{ Experiment 2 } \\
\cline { 2 - 4 } \cline { 6 - 8 } Location & $m$ & $b$ & $r_{\text {corr }}$ & & $m$ & $b$ & $r_{\text {corr }}$ \\
\hline Azimuth & 0.71 & -8.6 & .70 & & 0.72 & -6.8 & .75 \\
Elevation & 1.05 & -10.6 & .93 & & 0.92 & -9.3 & .95 \\
Distance & 1.06 & -0.1 & .85 & & 1.06 & -0.1 & .92 \\
\hline
\end{tabular}

Note-The transformed value of response $x$ in each case is $m x+b$, where $m$ is the slope and $b$ is the $y$-intercept. The correlation $\left(r_{\text {corr }}\right)$ between target and response locations is also showp. 
Table 12

Mean Responses (With Standard Deviations) for Forward-Facing and Profiled Small-Head Conditions

\begin{tabular}{|c|c|c|c|c|}
\hline \multirow[b]{2}{*}{ Error Type } & \multicolumn{2}{|c|}{ Forward-Facing } & \multicolumn{2}{|c|}{ Profiled } \\
\hline & $M$ & $S D$ & $M$ & $S D$ \\
\hline Azimuth† & $0.6^{\circ}$ & 11.6 & $-3.4^{\mathrm{o}}$ & 12.7 \\
\hline Elevation $\dagger$ & $7.0^{\circ}$ & 9.7 & $9.7^{\circ}$ & 9.1 \\
\hline Distance & $-1.8 \%$ & 26.4 & $-0.6 \%$ & 21.2 \\
\hline Angle & $15.9^{\circ}$ & 7.9 & $15.6^{\circ}$ & 7.7 \\
\hline Vector length* & $38 \%$ & 18 & $35 \%$ & 15 \\
\hline
\end{tabular}

Note-The ${ }^{*}$ and ${ }^{\dagger}$ indicate differences significant at the $p<.05$ and .001 levels, respectively (two-tailed $t$ test).

sponse methods indicated that a reversal had occurred, the responses were "corrected" by reflecting the subject response across the front-back plane, as has been discussed by Wightman and Kistler (1989).

\section{Results and Discussion}

The results were analyzed separately for stimuli in the front and rear hemispheres of the subject (trials with azimuth, greater than $-90^{\circ}$, and trials with azimuth, less than $-90^{\circ}$ ). The trials were about evenly divided between the front and the rear (208 front, 184 rear). The results can be summarized as follows (see Table 13).

1. In the front hemisphere, the errors with the DL response and an acoustic stimulus are much larger than the corresponding DL errors seen earlier with a visual stimulus. Specifically, the angle error of $12^{\circ}$ and the vector length error of $37 \%$ in Experiment 3 are both approximately double the values in Experiment $1\left(6^{\circ}\right.$ and $21 \%$; Table 2). For the SH method, however, the errors are approximately the same as those in Experiments 1 and 3. These results suggest that the response error from uncertainty about the location of the auditory target dominates the DL errors but that the inaccuracies inherent to the SH response method contribute substantially to its overall variability.

2 . The vector length and angle errors with the DL method and an auditory target (Experiment 3 ) are both comparable with the best performance seen for the SH response with a visual target (Experiment 2; see Table 10).
In other words, the combination of variability associated with auditory localization and the noise in the DL task is about the same as the noise for the $\mathrm{SH}$ response alone. Since the DL method is more accurate with an auditory stimulus than the other methods are with a visual stimulus, the superiority of the DL response in Experiment 1 cannot be explained by the hypothesis that the subjects were simply moving the response wand to the location that matched the visual image of the previously observed stimulus.

3. For each response method, performance in the front and rear hemispheres is similar, in terms of both mean errors and standard deviations. Although a few error measures do increase significantly (SH angle error is $22 \%$ higher in the rear hemisphere), overall differences are small. This result is surprising. A priori, one would expect the DL task to be substantially less accurate when the subject is forced to respond outside the visual field and at locations in the rear hemisphere that are relatively difficult to reach. However, these data indicate that average performance is virtually identical in the front and the rear hemispheres. Thus, any degradation associated with positioning the response sensor out of the visual field is small, as compared with the overall noisiness of the auditory localization task.

\section{CONCLUSIONS}

1. The DL response is markedly superior to any of the alternative response methods tested. When the DL response was the first response made with a visual target, the mean angle error was only $4^{\circ}$ and the mean vector length error was only $12 \%$. In contrast, the next-best response method produced angle and vector length errors roughly three times as large. DL was also the only response without significant biases for azimuth, elevation, and distance. Clearly, the DL response is the most appropriate for a near-field localization experiment.

2 . Replacing the visual target with an acoustic source approximately doubled the errors in the DL response method. The errors in the acoustic experiment were, however, approximately equal in the front and the rear hemi-

Table 13

Comparison of Response Accuracy in the Front and Rear Hemispheres for the Direct Location and Small-Head Responses in Experiment 3

\begin{tabular}{|c|c|c|c|c|c|c|c|c|}
\hline \multirow[b]{3}{*}{ Error } & \multicolumn{4}{|c|}{ DL } & \multicolumn{4}{|c|}{ SH } \\
\hline & \multicolumn{2}{|c|}{ Front } & \multicolumn{2}{|c|}{ Rear } & \multicolumn{2}{|c|}{ Front } & \multicolumn{2}{|c|}{ Rear } \\
\hline & $M$ & $S D$ & $M$ & $S D$ & $M$ & $S D$ & $M$ & $\overline{S D}$ \\
\hline$\overline{\text { Azimuth }}$ & $-4.9^{\circ}$ & 10.7 & $-0.1^{\circ}$ & 11.1 & $-5.7^{\circ}$ & 17.1 & $-14.8^{\circ}$ & 13.6 \\
\hline Elevation & $0.2^{\circ}$ & 11.4 & $4.4^{\circ}$ & 11.5 & $8.3^{\circ}$ & 13.2 & $1.5^{\circ}$ & 13.0 \\
\hline Distance & $11.9 \%$ & 32.5 & $8.9 \%$ & 29.7 & $-1.5 \%$ & 33.8 & $1.6 \%$ & 34.4 \\
\hline Angle & $11.9^{\circ}$ & 8.4 & $13.9^{\circ}$ & 8.0 & $16.9^{\circ}$ & 11.7 & $20.7^{\circ}$ & 9.6 \\
\hline Vector length & $37 \%$ & 24 & $38 \%$ & 21 & $43 \%$ & 23 & $48 \%$ & 24 \\
\hline
\end{tabular}

Note-Mean errors are shown separately for the front and rear hemispheres with the direct location (DL) and small-head (SH) response methods. The stimulus was the location of a short sound signal, a 125 -msec burst of white noise. 
spheres. Although it is possible that the DL response was noisier in the rear hemisphere than in the front hemisphere, it appears that the DL response errors in either hemisphere are small, as compared with the uncertainty in the auditory localization task.

3. Performances were similar for indirect location with a large or a small manikin head (the LH and SH methods) and with the verbal report of coordinates (the VR method), once the responses were corrected for overall biases. Angle errors ranged from $13.2^{\circ}$ to $16.5^{\circ}$, and vector length errors ranged from $35 \%$ to $39 \%$. Although the VR method was slightly more accurate, it was slower than the other response methods. The $\mathrm{SH}$ response was always at least as accurate as the $\mathrm{LH}$ response, indicating that scaling distance down by a factor of two did not adversely influence response accuracy.

4. Although variations in response biases across subjects were evident, bias trends common to all the subjects were substantial. In particular, a bias correction that depended only on response elevation improved performance at least $60 \%$ as much as a more complicated correction scheme based on the individual subject biases.

5 . When giving all four responses on each trial (Experiment 1 ), response accuracy was affected by response order only for the DL method, and not for any of the three indirect response methods ( $\mathrm{LH}, \mathrm{SH}$, and VR). The DL errors were about twice as large when it was the last response than they were when it was the first response.

6. Response errors in the $\mathrm{LH}$ and $\mathrm{SH}$ methods were highly correlated, implying that both responses may be derived from the same encoded memory of the target location. Correlations between the other response methods were small.

7. Once the responses were bias corrected, the errors were roughly independent of target location. Vector length error was negatively correlated with distance for all four response methods, but the correlation was relatively weak (average $r=-.28$ ).

8 . The SH response was not significantly different in angular error when the manikin head was profiled than it was when the head faced the same direction as the subject.
In summary, the DL method was superior to the three indirect response methods in terms of having the best overall accuracy, the smallest biases, and the shortest response times. These advantages promote DL as an attractive response metric for future localization studies. One caveat to this recommendation is that the present work utilized stimulus locations restricted to one side of the head. This restriction allowed positioning of the response sensor with a wand without the need for passing the wand from one hand to the other. Such passing or some other modification might be required if stimulus distances up to a meter at any angle about a subject were to be included for testing. Evaluations of such response modifications await future research.

\section{REFERENCES}

Butler. R., Levy, E., \& NeFF. W. (1980). Apparent distance of sounds recorded in echoic and anechoic chambers. Journal of Experimental Psychology, 6, 745-750.

COLEMAN. P. (1968). Dual role of frequency spectrum in determination of auditory distance. Journal of the Acoustical Society of America, 44, 631-632.

Durlach, N., \& Braida, L. (1969). Intensity perception: I. Preliminary theory of intensity perception. Journal of the Acoustical Society of America, 46, 372-383.

FOLEY, J. (1977). Effect of distance information and range on two indices of visually perceived distance. Perception, 6, 449-480.

Gilkey, R. H., Good, M. D., Ericson, M. A., Brinkman, J., \& StewART, J. M. (1995). A pointing technique for rapidly collecting localization responses in auditory research. Behavior Research Methods, Instruments, \& Computers, 27, 1-11

Makous. J., \& MiddeEbrooks. J. (1990). Two-dimensional sound localization by human listeners. Journal of the Acoustical Society of America, 87, 2188-2200.

MERShon, D., \& Bowers, J. (1979). Absolute and relative cues for the auditory perception of egocentric distance. Perception, 8, 311-322.

Soechting, J., \& Flanders, M. (1989). Sensorimotor representations for pointing to targets in three-dimensional space. Journal of Neurophysiology, 63, 582-592.

Wightman, F., \& Kistler, D. (1989). Headphone simulation of freefield listening: II. Psychological validation. Journal of the Acoustical Society of America, 85, 868-878.

Wightman, F., \& Kistler, D. (1992). The dominant role of lowfrequency interaural time differences in sound localization. Journal of the Acoustical Society of America, 91, 1648-1660.

\title{
APPENDIX \\ Coordinate System Definitions
}

\begin{abstract}
One of the key goals of this research was a comparison between judgments of location relative to the subject's own head and judgments of location relative to a manikin head. This requires a coordinate system that is consistent for both the subject's frame of reference and the manikin head's frame of reference. Three features common to both the human and the manikin head were used as the basis of this coordinate system: the openings of the left and right ear canals and the tip of the nose. The locations of these three features generate a coordinate system for each head centered at the midpoint of the interaural axis and with a horizontal plane approximately parallel with the floor. Specifically, head-referenced Cartesian axes were defined as follows: The $y$-axis of the coordinate system is the interaural axis of the head and is positive on the left side. The $x$-axis is the perpendicular bisector of the interaural axis passing closest to the tip of the nose and is positive for locations in
\end{abstract}




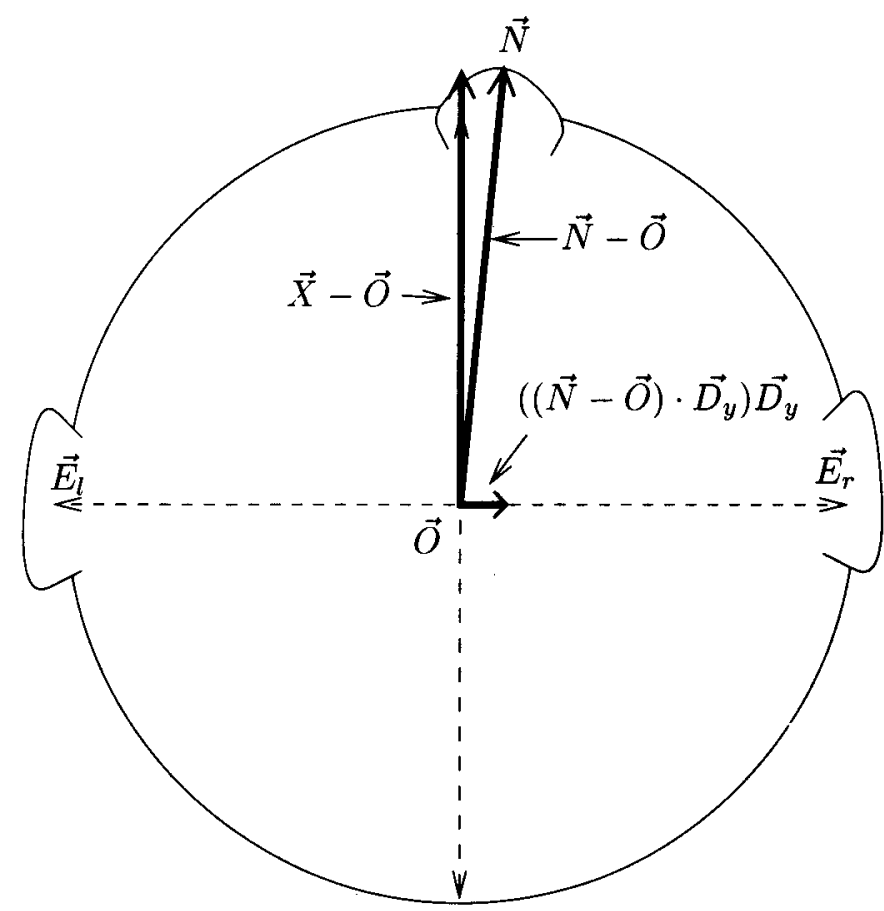

Figure A1. Definition of the $x$-axis. In this example, the vector $\vec{N}-\vec{O}$ is not perpendicular to the interaural axis. The projection of $\vec{N}-\vec{O}$ onto the $y$-axis, $\left\{\left[(\vec{N}-\vec{O}) \cdot \vec{D}_{Y}\right] \vec{D}_{Y}\right\}$, is subtracted from $\vec{N}-\vec{O}$ to yield the vector $\vec{X}-\vec{O}$, which is a perpendicular bisector of the interaural axis. Normalization of $\vec{N}-\vec{O}$ gives the directional cosines of the $x$-axis.

front of the head. The $z$-axis is perpendicular to both the $x$-axis and the $y$-axis and is positive above the head.

The reference points for these coordinate systems were measured with the 3SPACE tracker before each block of trials. The tracker provided the $x, y$, and $z$ locations of each position relative to the 3SPACE source, which was mounted on the chinrest. The locations of the left and right ears and the tip of the nose, represented as column vectors $\vec{E}_{1}, \vec{E}_{\mathrm{r}}$, and $\vec{N}$, were measured for the subject, the large manikin head, and the small manikin head with a 3SPACE tracker sensor. These locations were used to determine the origin and the directional cosines of the $x-, y$-, and $z$ axes for each of the three heads.

The origin of the coordinate system $\vec{O}$ is defined as the midpoint of the interaural axis,

$$
\frac{\vec{E}_{1}+\vec{E}_{\mathrm{r}}}{2}
$$

The interaural axis also defines the directional cosines of the $y$-axis,

$$
\vec{D}_{Y}=\frac{\vec{E}_{1}-\vec{E}_{\mathrm{r}}}{\left|\vec{E}_{\mathrm{l}}-\vec{E}_{\mathrm{r}}\right|} .
$$

The $x$-axis is defined by the tip of the nose, the origin $\vec{O}$, and the directional cosines of the $y$-axis $\vec{D}_{Y}$. Ideally, the $x$-axis should pass through the tip of the nose, but the vector $\vec{N}-\vec{O}$ is not necessarily perpendicular to the $y$-axis, as can be seen in Figure $\mathrm{Al}$. When this is true, the projection of $\vec{N}-\vec{O}$ onto the $y$-axis is subtracted from $\vec{N}-\vec{O}$, to determine the perpendicular bisector of the interaural axis that is closest to the tip of the nose. If we call this vector $\vec{X}-\vec{O}$,

$$
\vec{X}-\vec{O}=(\vec{N}-\vec{O})-\left[(\vec{N}-\vec{O}) \cdot \vec{D}_{Y}\right] \vec{D}_{Y},
$$

and the directional cosines of the $x$-axis are found by normalization 


$$
\vec{D}_{X}=\frac{\vec{X}-\vec{O}}{|\vec{X}-\vec{O}|}
$$

Finally, the directional cosines of the $z$-axis are obtained by taking the cross product of the $\vec{D}_{X}$ and $\vec{D}_{Y}$ vectors, so $\vec{D}_{Z}=\vec{D}_{X} \times \vec{D}_{Y}$.

The directional cosines can be used to convert the Cartesian coordinates of any location, measured relative to the head tracker, into the transformed coordinate system relative to the head. Let $\vec{S}$ be an $x y z$ location relative to the source of the 3SPACE tracker. First, the $x y z$ coordinates are moved relative to the center of the head by subtracting the origin of the transformed coordinate system $\vec{O}$ from $\vec{S}$. Then, the $x y z$ coordinates of the vector are projected onto the $x-, y$-, and $z$-axes of the transformed coordinate system by matrix multiplication

$$
\vec{S}_{T}=(\vec{S}-\vec{O})^{\prime} \cdot\left[\begin{array}{lll}
\vec{D}_{X} & \vec{D}_{Y} & \vec{D}_{Z}
\end{array}\right]
$$

where ' denotes matrix transposition. The resulting vector $\vec{S}_{\mathrm{T}}$ can be used to determine the azimuth, elevation, and distance of the target, relative to the coordinate system defined by one of the three heads, thereby allowing a direct comparison of all four response methods.

(Manuscript received January 28, 1998; revision accepted for publication November 18,1998 .) 